\title{
Use of acetazolamide in lithium-induced nephrogenic diabetes insipidus: a case report
}

\author{
Ricardo A Macau1,*, Tiago Nunes da Silva2,*, Joana Rego Silva1, \\ Ana Gonçalves Ferreira² and Pedro Bravo'1 \\ ${ }^{1}$ Nephrology Department, Hospital Garcia de Orta and 2Endocrinology Department, Hospital Garcia de Orta, \\ Almada, Portugal
}

Correspondence should be addressed to R A Macau or T N da Silva Email

ricardoandremacau@gmail. com or tiago_fcml@yahoo. com

\section{Summary}

Lithium-induced nephrogenic diabetes insipidus (Li-NDI) is a rare and difficult-to-treat condition. A study in mice and two recent papers describe the use of acetazolamide in Li-NDI in 7 patients (a case report and a 6 patient series). We describe the case of a 63-year-old woman with bipolar disorder treated with lithium and no previous history of diabetes insipidus. She was hospitalized due to a bowel obstruction and developed severe dehydration after surgery when she was water deprived. After desmopressin administration and unsuccessful thiazide and amiloride treatment, acetazolamide was administrated to control polyuria and hydroelectrolytic disorders without significant side effects. To our knowledge, this is the third publication on acetazolamide use in Li-NDI patients.

\section{Learning points:}

- Treatment of lithium-induced nephrogenic diabetes insipidus might be challenging.

- Vasopressin, amiloride and thiazide diuretics have been used in lithium-induced nephrogenic diabetes insipidus treatment.

- Acetazolamide might be an option to treat lithium-induced nephrogenic diabetes insipidus patients who fail to respond to standard treatment.

- The use of acetazolamide in lithium-induced nephrogenic diabetes insipidus must be monitored, including its effects on glomerular filtration rate.

\section{Background}

Diabetes insipidus (DI) is a rare condition in which patients excrete large quantities of diluted urine (up to 1 litre per hour in the most severe cases), leading to dehydration in the absence of adequate water intake. It can be either central (resulting from failure to synthesize or secrete vasopressin from the hypothalamus or posterior pituitary) or nephrogenic (resulting from kidney inability to respond to vasopressin). Nephrogenic diabetes insipidus (NDI) can result from genetic causes, such as mutations in the V2-vasopressin receptor (V2R) or the aquaporin-2 (AQP2) water channel, or acquired causes, such as lithium therapy. The latter is the most common form of NDI, and is known as lithium-induced NDI (Li-NDI) (1, 2, 3, $4,5,6,7)$. Lithium enters cells through several (sodium) transporters, but there is limited transportation out of cells, resulting in intracellular accumulation. Some controversy exists regarding the pathogenicity of intracellular lithium damage (8). One model proposes that intracellular lithium inhibits adenylyl cyclase in the collecting duct, disrupting the activation of vasopressin-sensitive transport proteins, thus resulting in polyuria and NDI (9). In addition, chronic lithium therapy decreases both AQP2 and urea 
transporter-A1 protein expression, further contributing to NDI (10). However, lithium-induced downregulation of AQP2 and development of NDI may occur independently of adenylyl cyclase activity (11).

The management of Li-NDI is challenging, even after lithium discontinuation. Thiazide diuretics, amiloride and reduced sodium intake have been used as standard therapy.

Thiazide diuretics block the sodium-chloride cotransporter (NCC) in the distal convoluted tubule and thus exaggerate the hypovolemia and increase an already activated renin-angiotensin-aldosterone system (RAAS) further. This mechanism stimulates proximal tubule sodium and water reabsorption resulting in less volume delivery to the distal nephron $(1,2,3,12,13,14)$. Other data suggested that thiazides' beneficial effect in lithiuminduced NDI may derive from inhibition of CA, both by direct vascular effects (15) and also anti-diuresis and alkalinization of urine in Li-NDI mice lacking NCC (14). Amiloride inhibits lithium entry into renal collecting duct cells through the epithelial sodium channel (ENaC) $(1,8$, $10,12)$. Non-steroidal anti-inflammatory drugs have been used but they are nephrotoxic $(1,2,3,5,8,12)$.

In an animal model of Li-NDI, acetazolamide (a CA) was as effective as the combination of hydrochlorothiazide and amiloride in reducing polyuria and increasing urine osmolality and AQP2 abundance. Acetazolamide also showed fewer side effects. In particular, the thiazide/ amiloride-treated mice developed hyponatraemia, hyperkalaemia, hypercalcaemia, metabolic acidosis and increased serum lithium concentrations, and these side effects were not observed in the acetazolamide-treated mice. Groot and coworkers concluded that the reduction in polyuria after acetazolamide treatment was partially caused by a tubular-glomerular feedback response and reduced glomerular filtration rate (GFR), which also contributed to the reduction in urinary prostaglandin E2 levels and to the amelioration of the Li-NDI (16).

Supported by this model, Gordon and coworkers recently reported the first case of acetazolamide treatment to a 49-year-old patient with severe Li-NDI (after elective surgery and water depriving), resistant to hydrochlorothiazide treatment and other measures. Following treatment with acetazolamide, daily urine output decreased, urinary osmolality increased and of serum sodium levels normalized. No major adverse effects were observed (17). Groot and coworkers recently published a series of mice and 6 human patients with Li-NDI. Two patients withdrew from the study due to side effects. In the remaining patients, acetazolamide did not increased urine osmolality or decreased urine output significantly. In mice, acetazolamide partially reversed lithium-induced polyuria and increased urine osmolality. The decrease in urine output in mice was associated to a decreased glomerular filtration rate (GFR), which warrants against acetazolamide application in first line without further studies (18).

\section{Case presentation}

A 63-year-old woman with bipolar disorder on long-term lithium therapy ( $400 \mathrm{mg} /$ tid for 8 years) and no other relevant medical history was admitted to the emergency room with diffuse abdominal pain and constipation for ten days. She had polydipsia (water intake estimated of 5 litre per day) and polyuria for years that had not been investigated. On admission, her clinical examination revealed abdominal distension. Laboratory values showed: mild anaemia (haemoglobin: $114 \mathrm{~g} / \mathrm{L}$; reference range (RR): 115-180 g/dL), mild renal insufficiency (creatinine: $1.3 \mathrm{mg} / \mathrm{dL}$;RR: $0.5-0.9 \mathrm{mg} / \mathrm{dL}$ ) and a normal sodium of $140 \mathrm{mmol} / \mathrm{L}$ (RR: 135-145). Abdominal CT showed a stenotic lesion in the cecum and ascending colon, a probable metastatic lesion in the liver and bowel distention. An urgent right hemicolectomy plus metastectomy and ileostomy were performed, and the patient was admitted to the surgical ward. Over the next two days, her mental status progressively worsened, alongside increasing polyuria (from 650 to 2800 to $3620 \mathrm{~mL} /$ day), increasing natremia (140 up to 171 and then up to $181 \mathrm{mmol} / \mathrm{L}$ ) and increasing serum osmolality (up to $379 \mathrm{mosmol} / \mathrm{kg}$; RR: 275-295). Urinary osmolality achieved a maximum value of 303 mosmol/ Kg (RR: 300-900). Lithium concentration was in the supra-therapeutic range $(1.46 \mathrm{mmol} / \mathrm{L} ; \mathrm{RR}: 0.6-1.2)$ and was stopped. She was diagnosed with Li-NDI and transferred to the intensive care unit (ICU) where she was ventilated for airway protection due to impaired consciousness. Standard therapy with 5\% IV dextrose, hydrochlorothiazide 50 plus amiloride $5 \mathrm{mg}$ and desmopressin $2 \mathrm{mg}$ IV bid was initiated. No significant reduction in the urine output was observed after each desmopressin treatment and on day 8 after surgery, the patient maintained polyuria $(4200 \mathrm{~mL} /$ day) and hypernatraemia (154 mmol/L, Fig. 1).

\section{Treatment}

Following the publication of a recent report of successful Li-ND treatment with acetazolamide (15), the drug was 


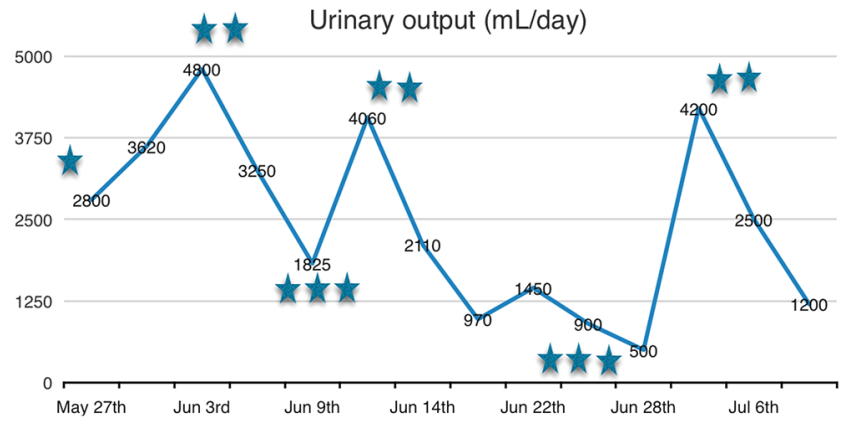

Figure 1

Daily urinary output during the admission. Surgery was performed on May 25th (*), acetazolamide was started on June 6th (**), stopped on June 9 th along with the other diuretics $(* * *)$, restarted in monotherapy on June 11 th $(* *)$, stopped again on June 27 th $(* * *)$ and restarted again on July 4 th $(* *)$

started at $500 \mathrm{mg}$ b.I.d. A progressive reduction of the urinary output was observed over the following days (4800-1825 mL/day) with a concomitant decrease in serum sodium $(154-138 \mathrm{mmol} / \mathrm{L})$ and a reduction of serum osmolality from 379 to $285 \mathrm{mosmol} / \mathrm{kg}$ (urine osmolality had decreased from 303 to 204 mosmol $/ \mathrm{kg}$ ) (Fig. 1). Acetazolamide and hydrochlorothiazide 50 plus amiloride $5 \mathrm{mg}$ were stopped after 6 days of acetazolamide treatment (day 14) with an increase in urinary output (1825-4060 mL/day) and serum sodium $(138-144 \mathrm{mmol} / \mathrm{L})$. These values decreased after restarting acetazolamide monotherapy on the 19th day after surgery (4600-2110 mL/day and $144-141 \mathrm{mmol} / \mathrm{L}$ respectively). A mild increase in urine osmolality was observed (from 252 to $263 \mathrm{mosmol} / \mathrm{kg}$ ).

\section{Outcome and follow-up}

The patient presented a ventilator-associated pneumonia, with isolation of Pseudomonas aeruginosa in a protected catheter sample, which was successfully treated with meropenem. Following improvement of the patient's mental status, she was weaned of ventilatory support and transferred to the surgery ward. The patient remained stable thereafter under acetazolamide treatment until day 33. Then, we identified a urinary tract infection (UTI) with an oliguric acute kidney injury (AKI). Fluids and antibiotic were administrated (meropenem to cover Extended-Spectrum Beta-Lactamases Producing Escherichia coli) and acetazolamide was stopped with resolution of the AKI. We also observed an increase in urinary output to $4200 \mathrm{~mL} /$ day, which normalized after reintroduction of acetazolamide $250 \mathrm{mg}$ bid $(1200 \mathrm{~mL} /$ day). The patient was subsequently discharged under

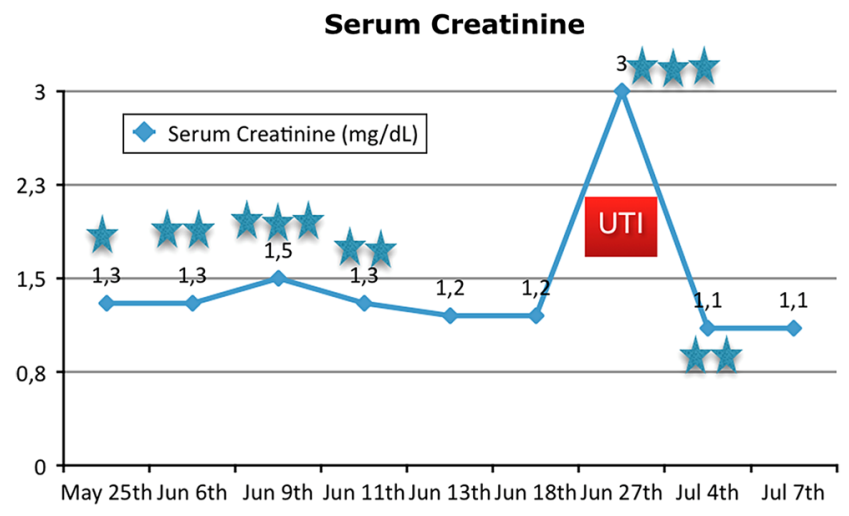

Figure 2

Creatinine as a marker of GFR during hospitalization. Surgery was performed on May 25th (*), acetazolamide was started on June 6th (**), stopped on June 9 th along with the other diuretics $(* * *)$, restarted in monotherapy on June 11 th $(* *)$, stopped again on June 27 th $(* * *)$ and restarted again on July 4 th $(* *)$. The patient was discharged on the 7 th of July. UTI and AKI were detected on June 27th.

acetazolamide treatment (day 44 after surgery), with no further polyuria on follow-up nor reported side effects. During treatment, neither significant hypokalaemia nor metabolic acidosis was detected. The patient reported drowsiness during hospitalization. It is unlikely that this was due to acetazolamide treatment.

\section{Discussion}

In a patient who presents with hypernatremic dehydration and diluted urine, a desmopressin test can help to distinguish between central DI or NDI. NDI is excluded with a urine osmolality $>800 \mathrm{mosmol} / \mathrm{kg}$ after desmopressin administration (1, 2, 3). In our patient that was chronically medicated with lithium and with polydipsia and polyuria habits (confirmed a posteriori), the diagnosis of Li-NDI can be made based on polyuria (diluted urine) and hypernatremic dehydration after water depriving and whose urinary osmolality and serum sodium level did not respond to desmopressin administration (1, 2, 3, 7). Hypotonic fluids were given intravenously (using 5\% dextrose in water). Infusion rate should only slightly exceed urine output in order to slowly normalize plasma natremia at rate of $<0.5 \mathrm{mmol} / \mathrm{L}$ per hour $(<10-12 \mathrm{mmol} / \mathrm{L}$ per day) considering the risk of cerebral oedema with a rapid decrease in plasma sodium $(1,3,5,7)$. Urine output can decrease quickly as dextrose in water will not effectively contribute to osmotic diuresis (despite being an osmolyte, dextrose is rapidly taken up by cells) (3). As discussed previously, the use of thiazide can decrease urine output. This reduction might be mediated to RAAS further activation, 
thus stimulating proximal tubule sodium and water reabsorption $(1,2,3,12,13,14)$. Other data showed that thiazides beneficial effect in lithium-induced NDI may derive from inhibition of $\mathrm{CA}$, both by direct vascular effects (15) and also anti-diuresis and alkalinization of urine in Li-NDI mice lacking NCC (14). An increase in AQP2 abundance at the apical membrane has also been demonstrated following the use of hydrochlorothiazide $(3,19)$. This contrasts with the earlier hypothesis that enhanced proximal sodium uptake leads to enhanced proximal water reabsorption and the balance between these mechanisms in these patients is yet to be clarified (3). On the other hand, functional studies suggested that water reabsorption downstream of the proximal tubule must also contribute to the anti-diuresis seen after prolonged thiazide treatment. Hydrochlorothiazide is able to directly increase the water permeability in inner medullary collecting ducts in mice and in vitro (19). Amiloride blocks the $\mathrm{ENaC}$ (decreasing sodium reabsorption in connecting tubules and collecting duct) and inhibits lithium entry into cells $(3,8,10,12)$. Discontinuation of lithium and the combination of thiazide diuretics and amiloride is successful in controlling polyuria in most of Li-NDI patients $(10,12,13)$.

Our patient remained polyuric under optimal doses of thiazide and amiloride treatment, with increased need of intravenous free water in order to compensate hypernatraemia. Based on the previous experience by Gordon et al. (17) and having in mind the data from Groot et al. (16), we decided to start acetazolamide. This led to a rapid decrease in urine output and correction of natremia. Acetazolamide reduces the glomerular filtration rate by means of tubulo-glomerular feedback and reduces renal prostaglandin secretion contributing to attenuate polyuria (17). There was a consistent relationship between use of acetazolamide and polyuria stabilization with negative and positive controls as seen in Fig. 1. We investigated possible side effects of acetazolamide (such as hypokalaemia or metabolic acidosis), which were not found. Similar to Groot and coworkers' most recent report, we observed only a mild and non-significant correction of urinary concentration impairment (18). It is difficult to associate the use of acetazolamide with the period of decreased GFR (day 33 after surgery) as there was a concomitant infection, and renal function remained stable otherwise (Fig. 2) but there might have been some degree of contribution. We cannot directly associate acetazolamide to some degree of drowsiness reported by the patient due to prolonged hospitalization (including ICU and ventilation).

The acetazolamide-induced reduction in urine output in our patient can be a direct consequence of the severe polyuria. Furthermore, the GFR reduction could explain the small increase in urine osmolality (similar to Gordon's report (17) and not verified in Groot's report (18) where only mild polyuria was observed). In patients with a chronic Li-NDI, sudden water deprivation (such as deprivation after surgery) might result in significant clinical deterioration; the use of acetazolamide might be useful in reducing polyuria through a GFR reduction.

Bearing in mind the recent data by Groot et al. (18), we cannot suggest acetazolamide as a first-line therapy. Nevertheless, we suggest this drug to be considered as an important second-line treatment in thiazide/amiloride refractory Li-NID, in selected cases and under tight GFR monitoring. Further studies assessing the safety of acetazolamide, particularly among patients with reduced or at risk of reducing GFR are required.

\section{Declaration of interest}

The authors declare that there is no conflict of interest that could be perceived as prejudicing the impartiality of the research reported.

\section{Funding}

This research did not receive any specific grant from any funding agency in the public, commercial or not-for-profit sector.

\section{Patient consent}

We have been informed that the patient died later after starting chemotherapy and we were not able to trace any relative.

\section{Author contribution statement}

Both co-first authors oversaw the conception and design of this case report, including reviewing of literature, article drafting and revising and approval of the final version to be published with equal level of contribution. Remaining authors participated with suggestions and corrections to the article (literature review and case report description) and took part in clinical decisions related to the patient. The authors acknowledge Andreia Leite for proofreading.

\section{References}

1 Bockenhauer D \& Bichet DG. Pathophysiology, diagnosis and management of nephrogenic diabetes insipidus. Nature Reviews Nephrology 201511 576-588. (https://doi.org/10.1038/ nrneph.2015.89) 
2 Boton R, Gaviria M \& Batlle DC. Prevalence, pathogenesis, and treatment of renal dysfunction associated with chronic lithium therapy. American Journal of Kidney Diseases 198710 329-345. (https://doi.org/10.1016/S0272-6386(87)80098-7)

3 de Groot T, Doornebal J, Christensen BM, Cockx S, Sinke AP, Baumgarten R, Bedford JJ, Walker RJ, Wetzels JFM, Deen PMT, et al. Lithium-induced NDI: acetazolamide reduces polyuria but does not improve urine concentrating ability. American Journal of Physiology: Renal Physiology 2017313 F669-F676. (https:/doi.org/10.1152/ ajprenal.00147.2017)

4 de Groot T, Sinke AP, Kortenoeven ML, Alsady M, Baumgarten R, Devuyst O, Loffing J, Wetzels JF \& Deen PM. Acetazolamide attenuates lithium-induce nephrogenic diabetes insipidus. Journal of the American Society of Nephrology 201627 2082-2091. (https://doi. org/10.1681/ASN.2015070796)

5 Gordon CE, Vantzelfde S \& Francis JM. Acetazolamide in lithiuminduced nephrogenic diabetes. New England Journal of Medicine 2016 375 2008-2009. (https://doi.org/10.1056/NEJMc1609483)

6 Kalra S, Zargar AH, Jain SM, Sethi B, Chowdhury S, Singh AK, Thomas N, Unnikrishnan AG, Thakkar PB, Malve H, et al. Diabetes insipidus: the other diabetes. Indian Journal of Endocrinology and Metabolism 201620 9-21. (https://doi.org/10.4103/22308210.172273)

7 Kishore BK \& Ecelbarger CM. Lithium: a versatile tool for understanding renal physiology. American Journal of Physiology: Renal Physiology 2013 304 F1139-F1149. (https://doi.org/10.1152/ ajprenal.00718.2012)

8 Klein JD, Gunn RB, Roberts BR \& Sands JM. Down-regulation of urea transporters in the renal inner medulla of lithium-fed rats. Kidney International 200261 995-1002. (https://doi.org/10.1046/j.15231755.2002.00210.x)

9 Li Y, Shaw S, Kamsteeg EJ, Vandewalle A \& Deen PM. Development of lithium-induced nephrogenic diabetes insipidus is dissociated from adenylyl cyclase activity. Journal of the American Society of Nephrology 200617 1063-1072. (https://doi.org/10.1681/ ASN.2005080884)

10 Loffing J. Paradoxical antidiuretic effect of thiazides in diabetes insipidus: another piece in the puzzle. Journal of the American Society of Nephrology 200415 2948-2950. (https://doi.org/10.1097/01. ASN.0000146568.82353.04)

11 Marples D, Christensen S, Christensen EI, Ottosen PD \& Nielsen S. Lithium-induced downregulation of aquaporin-2 water channel expression in rat kidney medulla. Journal of Clinical Investigation 1995 95 1838-1845. (https://doi.org/10.1172/JCI117863)

12 Moeller HB, Rittig S \& Fenton RA. Nephrogenic diabetes insipidus: essential insights into the molecular background and potential therapies for treatment. Endocrine Reviews 201334 278-301. (https:// doi.org/10.1210/er.2012-1044)

13 Pickkers P, Garcha RS, Schachter M, Smits P \& Hughes AD. Inhibition of carbonic anhydrase accounts for the direct vascular effects of hydrochlorothiazide. Hypertension 199933 1043-1048. (https://doi. org/10.1161/01.HYP.33.4.1043)

14 Saifan C, Nasr R, Mehta S, Acharya PS, Perrera I, Faddoul G, Nalluri N, Kesavan M, Azzi Y, El-Sayegh S, et al. Diabetes insipidus: a challenging diagnosis with new drug therapies. International Scholarly Research Notices: Nephrology 20132013 797620. (https://doi. org/10.5402/2013/797620)

15 Sands JM \& Bichet DG. Nephrogenic diabetes insipidus. Annals of Internal Medicine 2006144 186-194. (https://doi.org/10.7326/00034819-144-3-200602070-00007)

16 Sands JM \& Layton HE. Advances in understanding the urineconcentrating mechanism. Annual Review of Physiology 200476 387-409. (https://doi.org/10.1146/annurev-physiol-021113-170350)

17 Sands JM. Water, water everywhere: a new cause and a new treatment for nephrogenic diabetes insipidus. Journal of the American Society of Nephrology 201627 1872-1874. (https://doi.org/10.1681/ ASN.2015111223)

18 Shapiro M \& Weiss JP. Diabetes insipidus: a review. Journal of Diabetes and Metabolism 2012 S6 009. (https://doi.org/10.4172/2155-6156. S6-009)

19 Sinke AP, Kortenoeven ML, de Groot T, Baumgarten R, Devuyst O, Wetzels JF, Loffing J \& Deen PM. Hydrochlorothiazide attenuates lithium-induced nephrogenic diabetes insipidus independently of the sodium-chloride cotransporter. American Journal of Physiology: Renal Physiology 2014306 F525-F533. (https://doi.org/10.1152/ ajprenal.00617.2013)

Received in final form 19 December 2017

Accepted 16 January 2018 